\title{
Image Denoising Based on Human Visual Characteristics
}

\author{
Yuichi Nonaka \\ Media Systems Research \\ Department, Hitachi Ltd., \\ Tokyo, Japan \\ Graduate School of Engineering \\ Utsunomiya University, \\ Tochigi, Japan \\ yuichi.nonaka.zy@hitachi.com \\ Daisuke Yoshida \\ Media Systems Research \\ Department, Hitachi Ltd., \\ Tokyo, Japan. \\ daisuke.yoshida.cf@hitachi.com
}

\author{
Takashi Yokota \\ Graduate School of Engineering, \\ Utsunomiya University, Tochigi, \\ Japan \\ yokota@is.utsunomiya-u.ac.jp
}

\author{
Madoka Hasegawa \\ Graduate School of Engineering, \\ Utsunomiya University, \\ Tochigi, Japan \\ madoka@is.utsunomiya-u.ac.jp
}

\author{
Kanemitsu Ootsu \\ Graduate School of Engineering, \\ Utsunomiya University, \\ Tochigi, Japan \\ kim@is.utsunomiya-u.ac.jp
}

\begin{abstract}
In this paper, we propose a denoising method for color-noise reduction considering human visual characteristics for image noise. Our related work showed that there is an inverse correlation between contrast intensity of object edge signals and visual sensitivity of image noise. Based on the inverse correlation, our new method changes the noise-reduction level pixel-by-pixel in frequency domain using 2-dimensional discrete cosine transform (2D-DCT). We also considered the edge orientation of an object and the similarity between a target pixel and its neighboring pixels in determining the noise-reduction level. The evaluation results through image simulation showed that our proposed method is effective in achieving both high resolution and low noise.
\end{abstract}

\section{CCS Concepts}

- Computing methodologies $\rightarrow$ Computer graphics $\rightarrow$ Image manipulation $\rightarrow$ Image processing.

\section{Keywords}

Image denoising; DCT filtering; Human visual characteristics

\section{INTRODUCTION}

The use of vehicle cameras and surveillance cameras for assisting human visibility has been spreading rapidly. These cameras are required to capture clear images under any lighting environment and provide users with images with high visibility. However, images captured in dark environments include high levels of

\footnotetext{
Permission to make digital or hard copies of all or part of this work for personal or classroom use is granted without fee provided that copies are not made or distributed for profit or commercial advantage and that copies bear this notice and the full citation on the first page. Copyrights for components of this work owned by others than ACM must be honored. Abstracting with credit is permitted. To copy otherwise, or republish, to post on servers or to redistribute to lists, requires prior specific permission and/or a fee. Request permissions from Permissions@acm.org.
}

ICAIP 2017, August 25-27, 2017, Bangkok, Thailand

(C) 2017 Association for Computing Machinery.

ACM ISBN 978-1-4503-5295-6/17/08 ..\$15.00

DOI: https://doi.org/10.1145/3133264.3133267
Gaussian noise, which degrades visibility.

Various denoising methods that involve image processing have been investigated. Bilateral filtering (BLF) [13], for instance, is an edge-adaptive filtering-based method and is designed to both preserve the object edge signals and reduce the noise signals by adaptively changing the filter coefficient based on distance correlation and signal correlation. However, this method does not maintain the high resolution of images including object details. When object edge signals are smaller than noise signals, the object edge signals become blurred.

Local patch-based methods have been proposed, such as non-local means filtering (NLM) [4], block matching and 3D filtering (BM3D)[6], and Stein's unbiased risk estimator and guided piecewise linear estimation (S-PLE) [14]. With these methods, some image patches similar to the image patch including the denoising target pixel are selected from target pixel's neighboring area or from an image database. These patches are then blended with a weighted ratio, which is subject to their similarity. These methods enable to obtain low-noise and high-resolution images preserving the object edge signals of geometric shapes such as houses and automobiles composed of similar image patches. However, these methods do not denoise non-geometric shapes such as human faces, animals, and natural objects.

Frequency-based methods have also been proposed. For instance, the methods of WTMED [2] and WTJBF [1] use wavelet transform, and R-DCT [17] and RR-DCT [7] use discrete cosine transform (DCT). Signal separation in the frequency domain makes it easier to separate noise from the edge signal of an object. Therefore, frequency-based denoising methods are effective for achieving low-noise and high-resolution images. However, these methods still result in blurred images when applied to images with high levels of noise signals such as those captured in dark environments.

A denoising method using human visual characteristics is effective in solving the above problem. In our related study [9], we experimentally investigated human visual characteristics for Gaussian noise on a grayscale image and confirmed the effectiveness of a new frequency-based denoising method using 
human visual characteristics for grayscale images. By changing the noise-reduction level pixel-by-pixel based on the human visual characteristics in the frequency domain, it has been possible to improve subjective image quality. Furthermore, human visual characteristics for chroma noise were experimentally investigated [10]. We confirmed the effectiveness of filtering-based denoising methods using human visual characteristics for chroma signals. However, these methods still result in blurred images when applied to color images with high levels of noise signals.

To solve the problem described above, we propose a frequencybased denoising method using human visual characteristics for luminance and chroma signals. By changing the noise-reduction level pixel-by-pixel based on the human visual characteristics in the frequency domain, we can improve subjective image quality. In addition, by combining our method with a local patch-based approach, we can enhance the performance of denoising for geometrically shaped objects. We confirmed the effectiveness of our method through quantitative evaluation and subjective evaluation based on image simulation. The results indicate that our method leads to both lower-noise and higher-resolution images than traditional methods.

The rest of this paper is organized as follows. We explain the human visual characteristics through experimental confirmation in Section 2, the proposed method in Section 3, and evaluation results in Section 4. In Section 5, we conclude the paper.

\section{HUMAN VISUAL CHARACTERISTICS}

Human visual processing is controlled by several layers of the visual cortex in the brain [3][12], and various studies of human visual characteristics [5][8] have been conducted. It has been empirically shown that visual processing selectively highlights object signals in the event of a large quantity of informational transmission from the eyes to the brain. According to the principle of visual processing, Gaussian noise is not conspicuous on local image areas including high contrast intensity of object edge signals. For instance, as shown in Figure 1, even with the same level of noise signals, the visibility of noise varies depending on the contrast of the object edge signals. The noise is conspicuous on low edge-contrast areas. On the other hand, the noise is not conspicuous on high edge-contrast areas. By using the human visual characteristics for denoising, we can reduce conspicuous noise and maintain the contrast of object edge signals.

Our method involves changing the denoising level pixel-by-pixel based upon the contrast of the object edge signals. For instance, our method strengthens the denoising level in local image areas including conspicuous noise. Our method also weakens the denoising level in image areas including the high contrast intensity of object edges. This enables us to improve subjective image quality.

We have previously shown that the use of human visual characteristics for denoising is effective through experimental evaluation [9][10]. The relational expression, which represents the visibility of luminance noise, was derived through a subjective evaluation test [9]. The visibility of luminance noise is subject to the contrast intensity of object edge signals. The subjective luminance noise visibility $H n$ is expressed by Equation (1) using actual luminance noise intensity $E n$ and edge contrast intensity $E$ (described later in Section 3).

$$
H n=(1-0.0052 \times E) \times E n
$$

The equation shows the human visual characteristics in which $H n$ decreases linearly in accordance with $E$.

In the same way, the relation between visibility of chroma noise and edge contrast intensity was also derived [10]. The subjective chroma noise visibility Hcn is expressed by Equations (2) and (3) using the actual chroma noise intensity Ecn, luminance edge contrast intensity Eys, and chroma edge contrast intensity Ecs.

$$
\begin{gathered}
H c n=-0.0082 \times E y s \times E c n+E c n \\
H c n=-0.0051 \times E c s \times E c n+E c n
\end{gathered}
$$

These equations show the human visual characteristics in which $H c n$ decreases linearly in accordance with Eys. The Hcn is more affected by Eys than Ecs. For this reason, we use Equation (2) for denoising. Our proposed denoising method leads to low-noise and high-resolution images by changing the denoising level pixel-bypixel based on Equations (1) and (2).

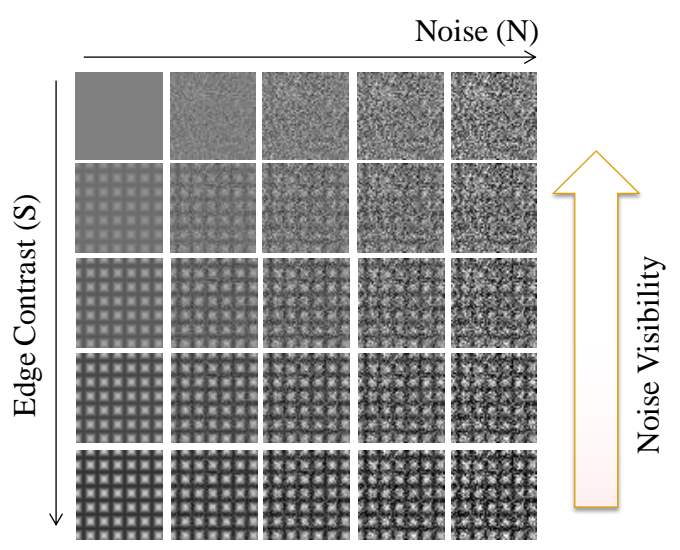

Figure 1. Correlation between levels of edge signals and noise

\section{PROPOSED DENOISING METHOD}

Figure 2 is a block diagram of our proposed denoising method. With this method, the noise is reduced by applying an $N \times N$ pixel filter using 2-dimensional discrete cosine transform (2D-DCT). The method consists of image signal separation ("step A"), three cascaded DCT filters for the luminance image, and three other cascaded DCT filters for the chroma image. Each filter consists of image-processing steps ("steps B through $F$ " for the $1^{\text {st }}$ and $2^{\text {nd }}$ filters, "steps B through $\mathrm{H}$ " for the $3^{\text {rd }}$ filter). A description of each step is follows.

\subsection{Image Signal Separation}

By Equation (4), an input image is separated into the luminance image $Y$ and chroma images $C 1, C 2$.

$$
\left(\begin{array}{c}
Y \\
C 1 \\
C 2
\end{array}\right)=\left(\begin{array}{ccc}
1 / \sqrt{3} & 1 / \sqrt{3} & 1 / \sqrt{3} \\
1 / \sqrt{6} & 0 & -1 / \sqrt{6} \\
1 / 3 \sqrt{2} & -2 / 3 \sqrt{2} & 1 / 3 \sqrt{2}
\end{array}\right)\left(\begin{array}{l}
R \\
G \\
B
\end{array}\right)
$$

\subsection{D-Discrete Cosine Transform}

Frequency components $F(k, l)$ of the $N \times N$ pixels by focusing on the target pixel are calculated using 2D-DCT expressed by Equation (5). 


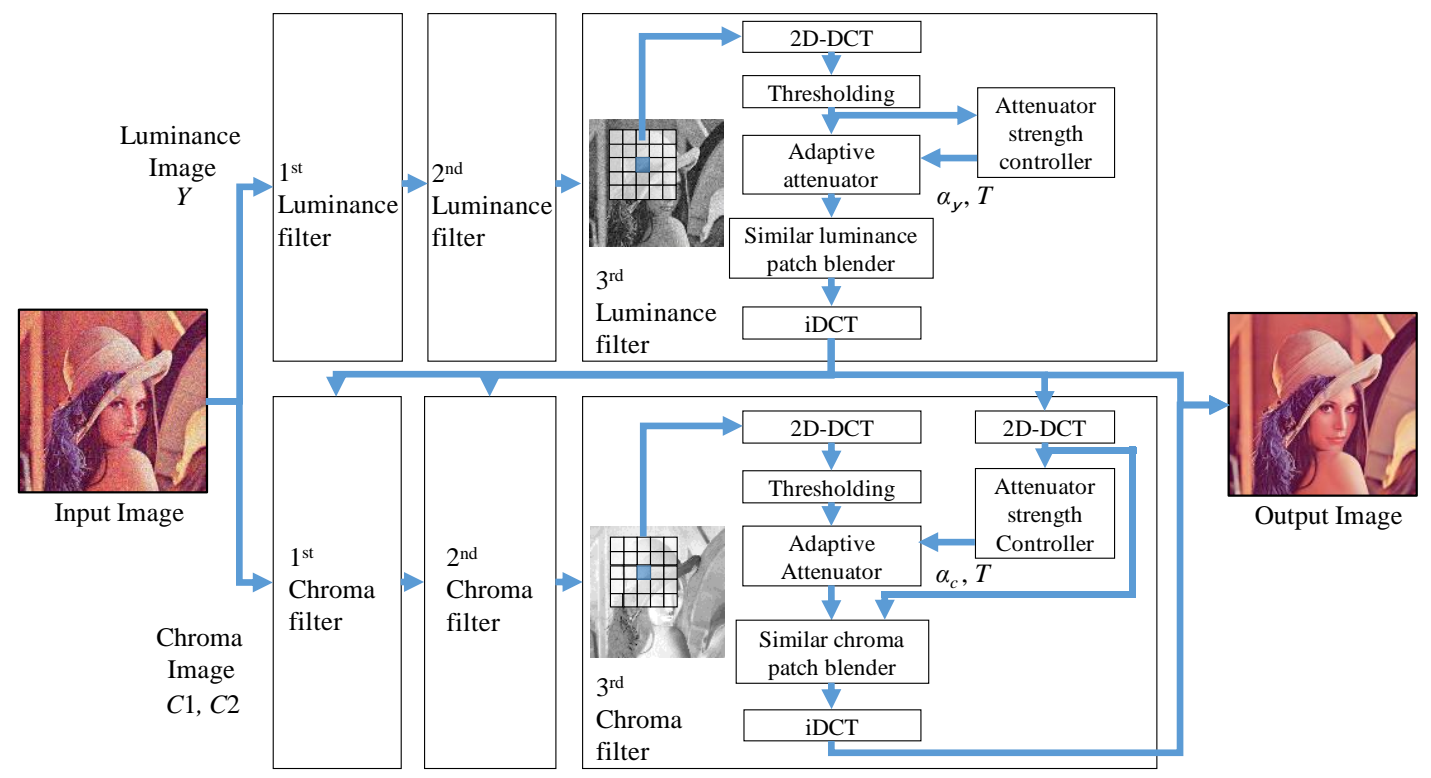

Figure 2. Block diagram of our method

$$
F(k, l)=\sum_{m=0}^{N-1} \sum_{n=0}^{N-1} f\left(x-\frac{N-1}{2}+m, y-\frac{N-1}{2}+n\right) \phi_{k}(m) \phi_{l}(n)
$$

Here, $(x, y)$ represents the coordinates of a target pixel for noise reduction, $f(x, y)$ represents the signal level of the pixel, $k$ represents the degree of horizontal frequency, and $l$ represents the degree of vertical frequency. The terms $\varphi(m)$ and $\varphi(n)$ are basis vectors of 2D-DCT, which are expressed by Equations (6) and (7), respectively.

$$
\begin{aligned}
& \phi_{k}(m)=\left\{\begin{array}{cc}
1 / \sqrt{2} & (k=0) \\
\sqrt{2 / N} \cos \{(2 m+1) k \pi / 2 N\} & (k=1,2, \ldots, N-1)
\end{array}\right. \\
& \phi_{l}(n)=\left\{\begin{array}{cc}
1 / \sqrt{2} & (l=0) \\
\sqrt{2 / N} \cos \{(2 n+1) l \pi / 2 N\} & (l=1,2, \ldots, N-1)
\end{array}\right.
\end{aligned}
$$

\subsection{Thresholding}

Thresholding is applied to each frequency component, except $F(0$, $0)$, to separate the edge signal and noise, as shown in Equations (8) and (9). Hereafter, $F(0,0)$ is referred to as a "direct current (DC) component" and $F(k, l)$ (both $k$ and $l$ are not 0 ) is referred to as an "alternative current (AC) component".

$$
\begin{aligned}
& F^{\prime}(0,0)=F(0,0) \\
& F^{\prime}(k, l)=\left\{\begin{array}{cc}
F(k, l)-T h & (F(k, l)>T h) \\
0 & (|F(k, l)| \leq T h) \\
F(k, l)+T h & (F(k, l)<-T h)
\end{array}\right.
\end{aligned}
$$

Here, Th represents a threshold value that is properly set to the signal level of the Gaussian noise. If an AC component exceeds Th and it is highly possible to be an edge signal of an object, it remains as it is. An AC component below Th, which is very likely to be noise, is reduced in this process.

\subsection{Adaptive Attenuator}

The AC components decrease using a noise visibility coefficient $\alpha$ and edge-orientation matrix $T(k, l)$. The decrease is expressed by Equations (10) and (11).

$$
\begin{aligned}
& F^{\prime \prime}(0,0)=F^{\prime}(0,0) \\
& F^{\prime \prime}(k, l)=(1-\alpha) T(k, l) F^{\prime}(k, l)
\end{aligned}
$$

Here, $\alpha$ is calculated for every pixel based on the human visual characteristics. It is computed using the attenuator strength controller. The $\alpha$ increases in areas where the luminance is flat; therefore, noise visibility is high. The AC components are largely suppressed (i.e. strengthening denoising level) in such areas.

The coefficient matrix $T(k, l)$ is also calculated pixel-by-pixel by using the attenuator strength controller, as described later. Each component of $T(k, l)$ corresponds to edge orientation and spatial frequency. Matrix $T(k, l)$ is determined based on the edge signals and orientation at a target pixel. If the horizontal frequency of an edge signal is smaller than its vertical frequency, the horizontal component of $T(k, l)$ is controlled to largely suppress the AC components (i.e. strengthened denoising level). The vertical component of $T(k, l)$ is controlled to maintain the AC components (i.e. weakened denoising level). Considering the edge orientation, this processing step leads to both denoising and edge preservation.

\subsection{Attenuator Strength Controller}

The $\alpha$ and $T(k, l)$ handled using the adaptive attenuator are calculated in accordance with each target pixel. The $\alpha$ is calculated individually for luminance and chroma signals, i.e., $\alpha_{y}$ applicable to luminance signals and $\alpha_{c}$ applicable to chroma signals. The $\alpha_{y}$ and $\alpha_{c}$ are derived using Equations (12) and (13), respectively, based on the human visual characteristics discussed in Section 2.

$$
\begin{aligned}
& \alpha_{y}=1-0.0052 \times E n \times E \\
& \alpha_{c}=1-0.0082 \times E n \times E
\end{aligned}
$$


The luminance $E$ in $N \times N$ pixels is expressed by Equation (14).

$$
E=\left\{\left(\sum_{k=0}^{N-1} \sum_{l=0}^{N-1}|F(k, l)|\right)-|F(0,0)|\right\} /(N \times N-1)
$$

At the luminance flat area, where there are high noise visibility and low $E$, the attenuator strength controller increases $\alpha$ to strengthen the denoising level. At the luminance edge area, where there are low noise visibility and high $E$, the attenuator strength controller decreases $\alpha$ to maintain the contrast intensity of an edge signal. In short, $\alpha$ fluctuates in accordance with the luminance $E$, which is based on the human visual characteristics.

Matrix $T(k, l)$ is a gain coefficient matrix for each frequency component. To calculate each component of $T(k, l)$ the horizontal edge intensity $E h$ and vertical edge intensity $E v$ within $N \times N$ pixels are first calculated as follows.

$$
\begin{aligned}
E h & =\sum_{k=0}^{N-1} \sum_{l=0}^{N-1}\{W h(k, l) *|F(k, l)|\} \\
E v & =\sum_{k=0}^{N-1} \sum_{l=0}^{N-1}\{W v(k, l) *|F(k, l)|\} \\
W h & =\left\{\begin{array}{ll}
1 & (k>l) \\
0 & (k \leq l)
\end{array}, \quad W v= \begin{cases}1 & (k<l) \\
0 & (k \geq l)\end{cases} \right.
\end{aligned}
$$

The $E h$ is a signal composed of a frequency component that mainly includes a horizontal edge component. The $E v$ is a signal composed of a frequency component that mainly includes a vertical edge component. Matrix $T(k, l)$ is calculated by Equations (18), (19), and (20).

$$
\begin{aligned}
& T(k, l)=\frac{E h}{E h+E v} \times H(k, l)+\frac{E v}{E h+E v} \times V(k, l) \\
& H(k, l)=1.25-(\arctan (l / k) \times 180 / \pi) / 90 \\
& V(k, l)=0.25+(\arctan (l / k) \times 180 / \pi) / 90
\end{aligned}
$$

Matrices $H(k, l)$ and $V(k, l)$ are fixed parameters expressed by Equations (19) and (20). Matrix $T(k, l)$ is calculated by blending $H$ and $V$ with the respective ratios of $E h$ and $E v$ expressed by Equation (18). In short, for example, when horizontal object edge signals exist within $N \times N$ pixels, $E h$ increases and $E v$ decreases. In this case, $T(k, l)$ is composed of a higher ratio of $H$ and lower ratio of $V$. As a result, $T(k, l)$ has filtering characteristics with which it maintains the horizontal frequency components of object edge signals and dampens the frequency components of the vertical edge signals. In short, the denoising level becomes high for frequency components containing only a low contrast intensity of object edge signals since resolution is hardly negatively affected. On the other hand, the denoising level becomes low for frequency components containing a high contrast intensity of object edge signals since noise is not conspicuous.

\subsection{Similar Luminance Patch Blender}

This step is applied to only the $3^{\text {rd }}$ luminance noise filter and blends a target pixel signal and its neighboring pixel signals in the frequency domain based on their similarity. Figure 3 shows an example in which a denoising target pixel is $(x, y)$. This function blends all the pixels in the $M \times M$ pixels search area. Equation (21) is used to calculate the difference between the patched pixel signals of $(x, y)$ and $(x-i, y-j)$ in the frequency domain. The terms $\triangle D C(x, y, i, j)$ and $\triangle A C(x, y, i, j)$ represent gaps of the DC and AC components between the above two patched pixels, respectively. These gaps are expressed by Equations (22) and (23).

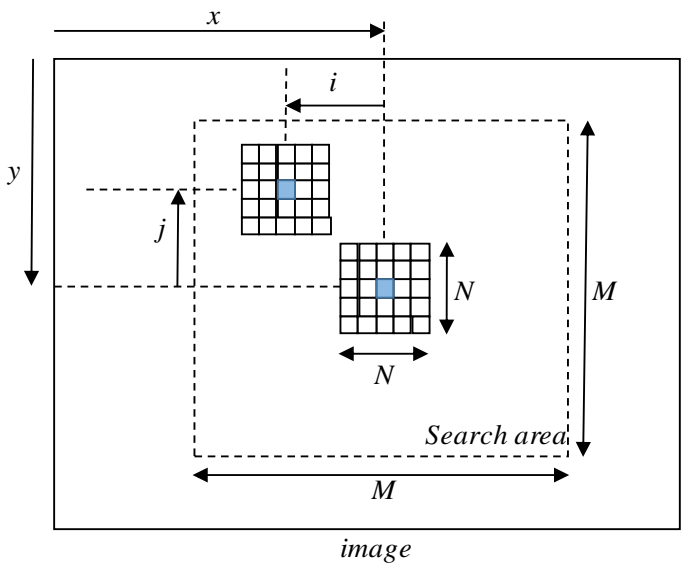

Figure 3. Similar luminance patch blender

$$
\begin{aligned}
& \Delta(x, y, i, j)=\frac{1}{N^{2}} \Delta D C(x, y, i, j)+\Delta A C(x, y, i, j) \\
& \Delta D C(x, y, i, j)=\left|F^{\prime \prime}(x, y, k=0, l=0)-F^{\prime \prime}(x-i, x-j, k=0, l=0)\right| \\
& \Delta A C(x, y, i, j)=\sum_{k=0}^{N-1} \sum_{l=0}^{N-1}\left|F^{\prime \prime}(x, y, k, l)-F^{\prime \prime}(x-i, x-j, k, l)\right|-\Delta D C(x, y, i, j)
\end{aligned}
$$

Equation (24) blends each frequency component by weighted ratios based on the similarity of $\Delta(x, y, i, j)$ calculated above. The terms $\sigma_{1}$ and $\sigma_{2}$ are parameters need to determine in accordance with noise levels.

$$
F^{\prime \prime}(k, l)=\frac{\sum_{i=-M / 2}^{M / 2} \sum_{j=-M / 2}^{M / 2} F^{\prime \prime}(i, j, k, l) \exp \left(-\frac{i^{2}+j^{2}}{2 \sigma_{1}^{2}}\right) \exp \left(-\frac{\Delta(x, y, i, j)^{2}}{2 \sigma_{2}^{2}}\right)}{\sum_{i=-M / 2}^{M / 2} \sum_{j=-M / 2}^{M / 2} \exp \left(-\frac{i^{2}+j^{2}}{2 \sigma_{1}^{2}}\right) \exp \left(-\frac{\Delta(x, y, i, j)^{2}}{2 \sigma_{2}^{2}}\right)}
$$

\subsection{Similar chroma patch blender}

This step is applied to only the $3^{\text {rd }}$ chroma noise filter. Equation (24) is applied respectively to a luminance signal $Y$ and chroma signals $C 1$ and $C 2$. The average of these three values is calculated as $\Delta(x, y, i, j)$ by Equation (24) for a chroma signal.

\subsection{Inverse Discrete Cosine Transform}

By the inverse DCT (iDCT) defined in Equation (25), a center pixel value, $f^{\prime}(x y)$ of $N \times N, f^{\prime}(x y)$, is calculated and designated as an output pixel signal after denoising.

$$
f^{\prime}(x, y)=\sum_{k=0}^{N-1} \sum_{l=0}^{N-1} F^{\prime \prime}(k, l) \phi_{k}\left(\frac{N-1}{2}\right) \phi_{l}\left(\frac{N-1}{2}\right)
$$


Table 1. Parameters of each method

\begin{tabular}{c|l}
\hline Method & \multicolumn{1}{|c}{ Parameter } \\
\hline BLF & Filter Size: $17 \times 17, \sigma_{l}=3.0, \sigma_{2}=97.5$ \\
\hline WTJBF & Filter Size: $3 \times 3, \sigma_{l}=3.0, \sigma_{2}=50.0, \mathrm{~J}=2$ \\
\hline NLM & Search Area: $17 \times 17$, Patch Size: $5 \times 5, \mathrm{~h}=20$ \\
\hline BM3D & Search Area: $17 \times 17$, Patch Size: $8 \times 8$ \\
\hline R-DCT & Patch Size: $16 \times 16$ \\
\hline RR-DCT & Patch Size: $16 \times 16$ \\
\hline S-PLE & $\mathrm{S}=2$ \\
\hline & $1^{\text {st }}$ filter: $\mathrm{N}=17, \mathrm{Th}(\mathrm{Y})=500, \mathrm{Th}(\mathrm{C} 1, \mathrm{C} 2)=865$ \\
Ours & $2^{\text {nd }}$ filter: $\mathrm{N}=9, \mathrm{Th}(\mathrm{Y})=300, \mathrm{Th}(\mathrm{C} 1, \mathrm{C} 2)=520$ \\
& $3^{\text {rd }}$ filter: $\mathrm{N}=5, \mathrm{Th}(\mathrm{Y})=100, \mathrm{Th}(\mathrm{C} 1, \mathrm{C} 2)=150$, \\
$\mathrm{M}=11, \sigma_{l}=2.0, \sigma_{2}=80.0$
\end{tabular}

\section{EXPERIMENTS}

We conducted comparative experiments of image quality with several denoising methods on the basis of an image simulation. First, we selected 20 images from the standard image database (SIDBA) [11] and generated test images by adding Gaussian noise with standard deviation $\sigma=50$ to each image. Next, we obtained noise-reduced images for the test images by using traditional methods (NLM [4], WTJBF [1], BLF [13], R-DCT [17], BM3D [6], RR-DCT [7], and S-PLE [14]) and our proposed method. Table 1 shows the parameters of each method. The parameters of our method were experimentally determined. The others are recommended values described in each of their respective papers.

We conducted a quantitative evaluation of the images when applying each denoising method by using a quantitative index of image quality, i.e., structural similarity (SSIM) [15]. In terms of evaluating subjective image quality, the SSIM index is superior to the PSNR index. Table 2 lists the results of SSIM evaluation. The SSIM represents the value of visual similarity between the original image and noise-reduced image. The higher the SSIM is, the higher the similarity between the original image and noisereduced images. This indicates that a method results in highresolution and low-noise images. Our method achieved the highest SSIM for 12 out of 20 images and close to the highest SSIM for 4 images (Peppers, Tree, House2, and Splash). These 16 images consist of both low edge contrast areas and high contrast areas. With our method, changing the noise-reduction level pixel-bypixel based on edge contrast intensity led both low noise and high resolution for these images. However, three images (Aerial, Couple, Girl) consisted mostly of low edge contrast areas, and one image (Mandrill) consisted mostly of high edge contrast areas. The approach of changing the noise reduction level does not work effectively due to small variation in edge contrast intensity.

We conducted a subjective evaluation test on noise and resolution levels by showing 8 participants the same 20 images as mentioned above to which each method was applied. We asked the participants to score each image from 1 to 5 point, totaling a maximum of 100 points. Figure 4 shows the average points for the 20 images by the 8 participants. The vertical axis is the sum of the average points for each image based on our subjective evaluation results. It shows that, a higher bar indicates better image quality judged by the participants. Our proposed method earned the highest evaluation.

Figure 5 shows an example of the image-simulation results. We confirmed that our method could reduce color Gaussian noise on a flat area that included conspicuous noise and maintained the details of the edge signals on the edge areas. This was confirmed by there being no blurring on the surrounding spots of a bird's eye, which is observed with traditional methods' output images. This effect occurs by changing the denoising level pixel-by-pixel based on human visual characteristics and changing the frequency for noise reduction subject to the edge orientation. Our method also enables the reduction of color noise, maintaining the edge of the bird's stomach and beak portions. These areas include similar neighboring pixels, and these effects are observed by a weighted ratio of blending a target pixel and its neighboring pixels based on patched-image similarity. With our method, we confirmed that it is possible to achieve both low noise and high resolution, which are problems with traditional methods.

Finally, as a result of measuring the execution speed of our proposed method with $256 \times 256$ pixel image samples as input, the average processing time was 12.89 secs when executed five times on a PC with Corei7-5960X, 16-GB RAM and Windows 8.1. To provide high visibility images to users in real time using our method, it will be necessary to improve its processing speed.

\section{CONCLUSION}

We proposed a denoising method for reducing the Gaussian noise of colored images. The features of our method are as follows.

- By using 2D-DCT, our proposed method separates object edge signals and noise signals then reduces color noise by damping the ACs in the frequency domain.

- Based on human visual characteristics, our method reduces noise by changing its reduction level pixel-by-pixel in the frequency domain.

- Based on the ratio of horizontal edge contrast intensity and vertical edge contrast intensity, our method reduces color noise by changing its reduction level pixel-by-pixel, in accordance with each factor of 2D-DCT.

- Finally, our method blends a target pixel and its neighboring pixels in the frequency domain based on the similarity of patched images.

Based on the quantitative evaluation of images by SSIM, our proposed method achieved the highest SSIM for 12 out of 20 images. In addition, our proposed method earned the highest evaluation in the subjective image quality evaluation. We confirmed that our method leads to both low-noise and highresolution images.

For future work, it will be necessary to improve the processing speed of our method. 
Table 2. SSIM values of original image

\begin{tabular}{cccccccccc}
\hline Image & Noisy & BLF & WTJBF & NLM & BM3D & R-DCT & RR-DCT & S-PLE & Ours \\
\hline Aerial & 0.233 & 0.457 & 0.445 & 0.456 & 0.483 & 0.482 & 0.524 & $\mathbf{0 . 6 0 9}$ & 0.489 \\
Airplane & 0.188 & 0.524 & 0.702 & 0.738 & 0.782 & 0.768 & 0.763 & 0.797 & $\mathbf{0 . 7 9 9}$ \\
Balloon & 0.080 & 0.420 & 0.702 & 0.622 & 0.750 & 0.744 & 0.657 & 0.741 & $\mathbf{0 . 7 9 3}$ \\
Couple & 0.125 & 0.465 & 0.525 & 0.483 & 0.562 & 0.542 & 0.557 & $\mathbf{0 . 6 8 5}$ & 0.521 \\
Earth & 0.151 & 0.473 & 0.659 & 0.590 & 0.687 & 0.711 & 0.677 & 0.716 & $\mathbf{0 . 7 1 7}$ \\
Girl & 0.126 & 0.503 & 0.625 & 0.596 & 0.669 & 0.655 & 0.648 & $\mathbf{0 . 6 9 5}$ & 0.652 \\
Girl2 & 0.089 & 0.417 & 0.748 & 0.664 & 0.834 & 0.827 & 0.704 & 0.840 & $\mathbf{0 . 8 8 5}$ \\
Girl3 & 0.145 & 0.496 & 0.719 & 0.669 & 0.796 & 0.776 & 0.734 & 0.756 \\
House & 0.139 & 0.475 & 0.675 & 0.683 & 0.754 & 0.744 & 0.696 & 0.747 & $\mathbf{0 . 8 0 1}$ \\
JellyBeans & 0.090 & 0.470 & 0.811 & 0.802 & 0.883 & 0.865 & 0.772 & 0.872 \\
JellyBeans2 & 0.128 & 0.491 & 0.800 & 0.786 & 0.863 & 0.837 & 0.762 & 0.837 & $\mathbf{0 . 9 0 6}$ \\
Mandrill & 0.290 & 0.484 & 0.427 & 0.498 & 0.509 & 0.535 & 0.569 & $\mathbf{0 . 6 4 9}$ & 0.579 \\
Parrots & 0.140 & 0.500 & 0.707 & 0.692 & 0.783 & 0.775 & 0.723 & 0.781 & $\mathbf{0 . 8 0 9}$ \\
Peppers & 0.186 & 0.521 & 0.690 & 0.675 & $\mathbf{0 . 7 4 9}$ & 0.722 & 0.715 & 0.732 & 0.739 \\
Sailboat & 0.216 & 0.534 & 0.704 & 0.676 & 0.748 & 0.754 & 0.745 & 0.753 & $\mathbf{0 . 7 5 7}$ \\
Tiffany & 0.145 & 0.540 & 0.658 & 0.623 & 0.702 & 0.680 & 0.678 & 0.703 & $\mathbf{0 . 7 2 0}$ \\
Tree & 0.263 & 0.542 & 0.635 & 0.682 & 0.712 & 0.707 & 0.713 & $\mathbf{0 . 7 3 9}$ & 0.722 \\
House2 & 0.188 & 0.504 & 0.658 & 0.664 & 0.736 & $\mathbf{0 . 7 4 7}$ & 0.717 & 0.747 & 0.738 \\
Lenna & 0.119 & 0.484 & 0.680 & 0.654 & 0.739 & 0.743 & 0.693 & 0.721 & $\mathbf{0 . 7 5 6}$ \\
Splash & 0.089 & 0.499 & 0.743 & 0.743 & $\mathbf{0 . 8 0 2}$ & 0.794 & 0.749 & 0.765 & 0.800 \\
\hline
\end{tabular}

\section{Cumulative scores}

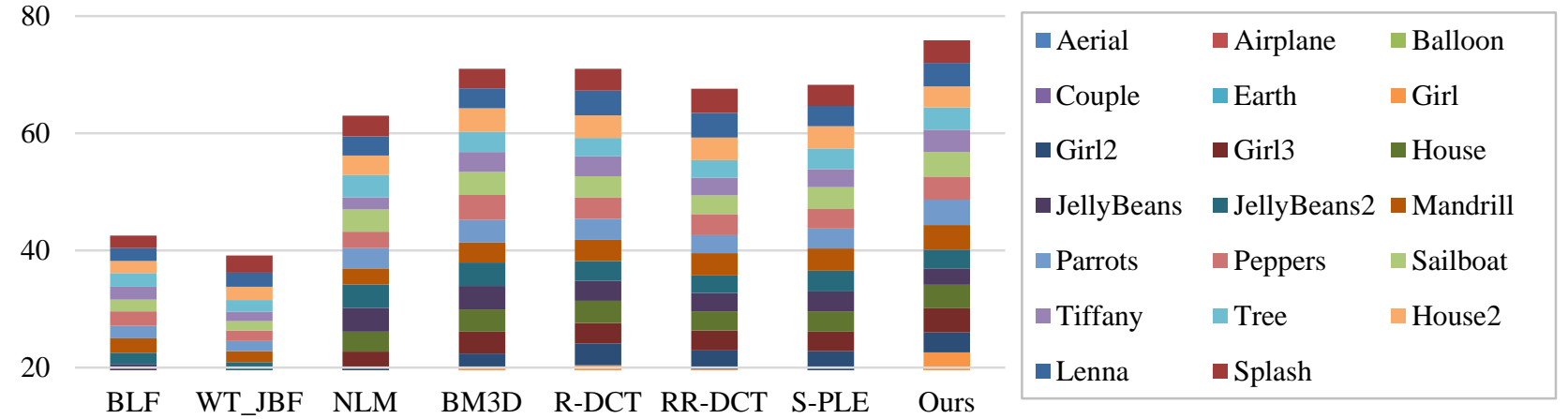

Figure 4. Experimental results of image quality through subjective evaluation

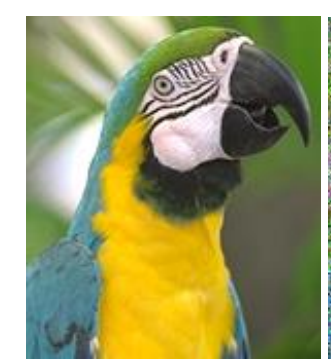

(a) original image

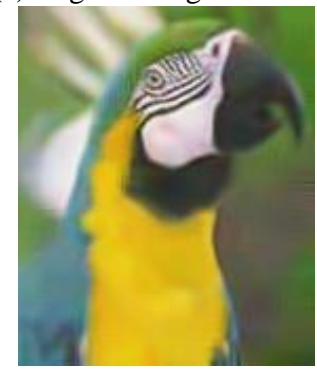

(f) $\mathrm{BM} 3 \mathrm{D}[6]$

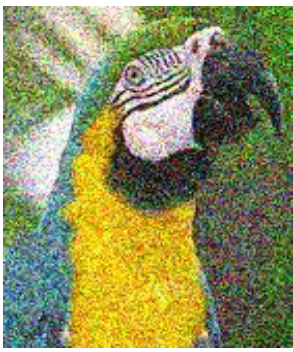

(b) noisy image $(\sigma=50)$

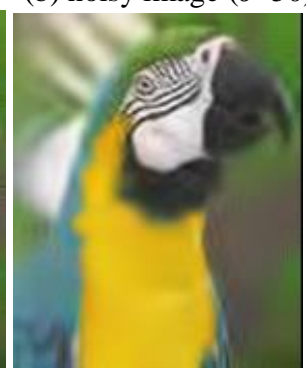

(g) R-DCT [17]

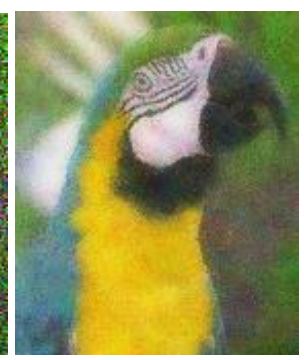

(c) BLF [13]

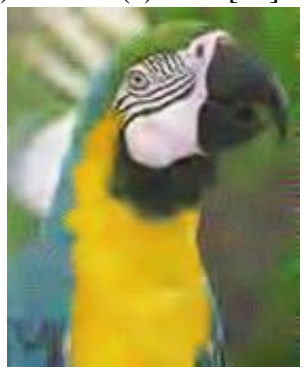

(h) RR-DCT [7]

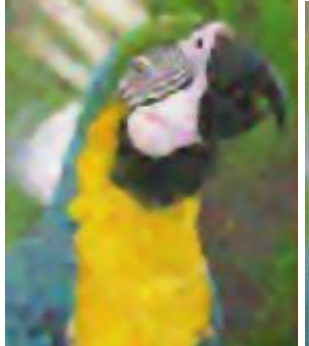

(d) WTBLF [1]
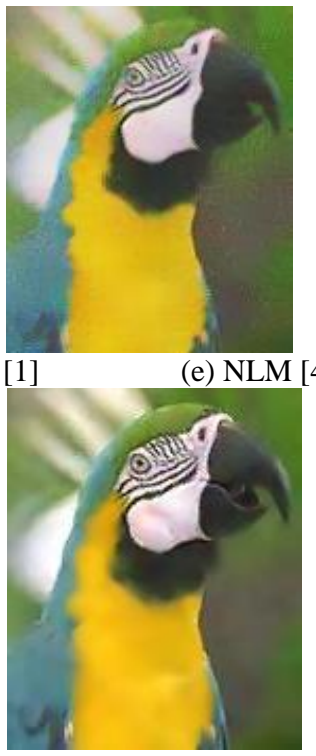

(i)S-PLE [14]

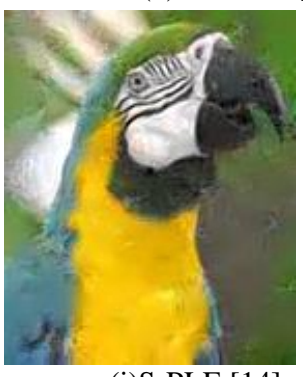

Figure 5. "Parrots": Input Image and Noise-Reduced Images by using each method

(j) our method 


\section{REFERENCES}

[1] Bibina, V. C. and Viswasom, S. 2012. Adaptive wavelet thresholding \& joint bilateral filtering for image denoising. 2012 Annual IEEE India Conference (INDICON). DOI=http://doi.org/10.1109/indcon.2012.6420781.

[2] Boyat, A. and Joshi, B. K. 2013. Image denoising using wavelet transform and median filtering. 2013 Nirma University International Conference on Engineering (NUiCONE). DOI=http://doi.org/10.1109/nuicone.2013.6780128.

[3] Brodmann, K. 1909. Vergleichende Lokalisationslehre der Großhirnrinde: in ihren Prinzipien dargestellt auf Grund des Zellenbaues. Leipzig, Barth.

[4] Buades, A. Coll, B., and Morel, J. M. 2005. A Non-Local Algorithm for Image Denoising. 2005 IEEE Computer Society Conference on Computer Vision and Pattern Recognition (CVPR'05).

DOI=http://doi.org/10.1109/cvpr.2005.38.

[5] Budisantoso, T. et al. 2012. Mechanisms Underlying Signal Filtering at a Multisynapse Contact. Journal of Neuroscience. 32, 7, 2357-2376.

DOI=http://doi.org/10.1523/jneurosci.5243-11.

[6] Dabov, K. et al. 2006. Image denoising with block-matching and 3D filtering. Image Processing: Algorithms and Systems, Neural Networks, and Machine Learning. DOI=http://doi.org/10.1117/12.643267.

[7] Fujita, S. et al. 2015. Randomized redundant DCT. SIGGRAPH ASIA 2015 Technical Briefs on - SA '15. (2015). DOI=http://dx.doi.org/10.1145/2820903.2820923.

[8] Kuboki, R. and Shitara M. 2013. Experimental System Architecture Affected by Visual Noise in Visual Recognition. Tsukuba Journal of Biology. 12, 69. (in Japanese)

[9] Nonaka, Y. and Yoshida, D. 2015. Edge Adaptive Noise Reduction Algorithm Based on Human Visual
Characteristics. The Journal of The Institute of Image Information and Television Engineers. 69, 3 (2015), 98-105. DOI=http://doi.org/10.3169/itej.69.j98. (in Japanese)

[10] Nonaka, Y. Yoshida, D. and Hirooka, S. 2015. Color Noise Reduction Algorithm Based on Human Visual Characteristics, IEICE Technical Report. 39, 16 (2015), 9-12. (in Japanese)

[11] Onoe, M. et al. 1979. SIDBA-Standard Image Data Base. Multidimensional Image Processing Center Report 79 , Institute of Industrial Science, University of Tokyo.

[12] Sato, H. and Katsuyama, N. 1993. Functional Organization and Roles of the Primary Visual Cortex. Japanese Orthoptic Journal. 21, (1993), 154-160.

DOI=http://doi.org/10.4263/jorthoptic.21.154.

[13] Tomasi, C. and Manduchi, R. 1998. Bilateral filtering for gray and color images. Sixth International Conference on Computer Vision (IEEE Cat. No.98CH36271). (1998). DOI=http://doi.org/10.1109/iccv.1998.710815.

[14] Wang, Y. Q. and Morel, J. M. 2013. SURE Guided Gaussian Mixture Image Denoising. SIAM Journal on Imaging Sciences. 6, 2, 999-1034. DOI=http://doi.org/10.1137/120901131.

[15] Wang, Z. et al. 2004. Image Quality Assessment: From Error Visibility to Structural Similarity. IEEE Transactions on Image Processing. 13, 4, 600-612.

DOI=http://doi.org/10.1109/tip.2003.819861.

[16] Yamamoto Y., Activate Human Brain by Tremor, $7^{\text {th }}$ group session of complex phenomenon engineering, National Institute of Advanced Industrial Science and Technology, Tsukuba. (2002). (in Japanese)

[17] Yu, G. and Sapiro, G. 2011. DCT image denoising: a simple and effective image denoising algorithm. Image Processing On Line. 1, DOI=http://doi.org/10.5201/ipol.2011.ys-dct. 